\title{
Utilization of Preventive Therapy in Korean Migraine Patients
}

\author{
Yewon Kim, Susin Park, Eonjeong Kim, and Nam Kyung Je* \\ College of Pharmacy, Pusan National University, Busan 46241, Republic of Korea \\ (Received December 10, $2020 \cdot$ Revised January 21, $2021 \cdot$ Accepted February 10, 2021)
}

\begin{abstract}
Background: Migraine is a common neurological disorder that affects the quality of life and causes several health problems. Preventive migraine treatment can reduce migraine frequency, headache severity, and health care costs. This study aimed to estimate the utilization of migraine preventive therapy and associated factors in eligible patients. Methods: We studied 534 patients with migraine who were eligible for migraine preventive therapy using 2017 National Patient Sample (NPS) data from the Health Insurance Review and Assessment Service (HIRA). We estimated the migraine days by calculating the monthly average number of defined daily dose (DDD) of migraine-specific acute drug. Patients with a monthly average number of DDD of 4 or more were considered as subjects for preventive treatment. Chi-square test and multiple logistic regression analysis were used to determine the association between the preventive therapy and the influencing variables. Results: Less than half of the eligible patients for prophylaxis $(n=234,43.8 \%$ ) were prescribed preventive therapy. Multiple logistic regression results show that migraine preventive therapy was influenced by age, the type of migraine, and some comorbidities. Patients over the age of 50 tend to receive less prophylactic treatment than under the age of 40 . On the other hand, migraine patients with epilepsy or depression were more likely to receive preventive therapy. Sumatriptan was the most preferred medication for acute treatment, and propranolol was the most commonly prescribed drug for prevention. Conclusions: More than half of the patients who were candidates for migraine prophylaxis were not receiving suitable preventive treatment. Positive factors affecting the use of migraine prevention were the presence of comorbidities such as epilepsy and depression.
\end{abstract}

KEYWORDS: Migraine, prophylaxis, migraine days, defined daily dose

Migraine is a common neurological disorder that occurs in approximately $12 \%$ of adults in western countries and can significantly impair the quality of life of the patients. ${ }^{1,2)}$ In Korea, $6.1 \%$ of the population suffers from migraine headaches at least once a year, and women roughly have three times higher incidence of migraine than men. It is most frequent in women aged 40-49 years and men aged 19-29 years. ${ }^{3}$ )

This neurological disorder has a broad range of severity and symptoms; it is a unilateral, pulsatile pain, and aggravated by routine physical activity. ${ }^{4)}$ In addition, migraine is accompanied by one or more additional symptoms such as nausea, vomiting, abdominal pain, dizziness, photophobia, and phonophobia. ${ }^{4)}$ According to the International Classification of Headache Disorders 3rd edition (ICHD-3), the two major subtypes of migraine are migraine without aura (MWoA) and migraine with aura (MWA), ${ }^{5)}$ and they are not completely distinct and exclusive. $^{5)}$

Appropriate acute therapy for migraine headaches can abort migraine attacks and suitable preventive treatment can reduce migraine frequency, headache severity, and health care costs. $\left.{ }^{6}\right)$ Acute treatments of migraines include serotonin receptor agonists (triptans), ergot alkaloids, non-steroidal anti-inflammatory drugs (NSAIDs), acetaminophen, and opioids. ${ }^{78}$ The American Academy of Neurology (AAN) has published guidelines for preventing migraines in 2012. ${ }^{9)}$ The AAN guidelines recommend metoprolol, propranolol, timolol, valproic acid/valproate/ divalproex, topiramate with strong evidence, and atenolol, nadolol, amitriptyline, venlafaxine with moderate evidence. ${ }^{9)}$

*Correspondence to: Nam Kyung Je, Pusan National University, College of Pharmacy, 2 Busandeahark-ro 63 beon-gil, Geumjeong-Gu, Busan 46241, Republic of Korea

Tel: +82-51-510-2802, Fax: +82-51-513-6754

E-mail: jenk@pusan.ac.kr 
The purpose of this study was to (1) examine the utilization of migraine preventive therapy in eligible patients, (2) determine the factors influencing the use of preventive therapy, and (3) assess the frequency of drugs prescribed for migraine-specific acute therapy and preventive therapy among Korean migraine patients.

\section{Methods}

\section{Data Source}

We utilized the National Patient Sample data from the Health Insurance Review and Assessment Service in 2017 (HIRA-NPS-2017-0014). NPS data is health insurance claims data of almost 1 million patients and includes patients' diagnosis, procedures, surgical history, medications, institutional information, etc. ${ }^{10)}$

The patient's diagnosis was identified using The Korean Classification of Diseases, 7th edition (KCD-7) code. KCD code is the Korean version of the International Classification of Diseases (ICD) code. There are three types of health insurance in Korea: The National Health Insurance (NHI), Medical Aid (MedAid), and the Patriots \& Veterans Insurance (PVI) plan. Approximately 97\% of Koreans are covered by NHI, about $3 \%$ of people are covered by MedAid, and $0.5 \%$ of people by the PVI plan. The drug codes (Appendix A) were used to extract information on migraine-specific acute and preventive therapy utilization. This study was approved by the Institutional Review Board of the Pusan National University (PNU IRB/2019_126_HR).

\section{Study Population}

Migraine patients with a KCD-7 code of G43 were extracted. The patients were further classified into MWoA (G430) and MWA (G431) using KCD-7 codes. In case a patient received both diagnoses at different times, the patient was labeled with the MWA type. The patients whose migraine subtype were not specified, were categorized into "the other migraine type". We grouped the patients by age into " $<40$ years", " $40-49$ years" and " $\geq 50$ years", and by insurance into NHI and MedAid/PVI group.

The KCD-7 code was used to identify the following comorbidities: epilepsy, depressive disorders, insomnia, hypertension, dyslipidemia, diabetes mellitus, osteoarthritis, rheumatoid arthritis, asthma, and Thyroid gland disorders (Appendix A). The comorbidities were referred to as common diseases occurring in more than $5 \%$ of migraine patients according to a previous study. ${ }^{11)}$

\section{Candidates for migraine prophylaxis}

Based on the previous studies and clinical trials, we considered patients with more than four migraine days per month as candidates for preventive treatment. ${ }^{4,12,13)}$ To estimate each patient's migraine days, the defined daily dose (DDD) was used. DDD is an average maintenance dose of a medication used by adults, which allows the drug used to be expressed in a standardized unit. ${ }^{14)}$ We estimated how many DDD of each migraine-specific medication was prescribed to a patient per month and summed them up. One DDD was regarded as one migraine day.

\section{Medication utilization of migraine-specific acute and preventive treatment}

Migraine-specific acute therapies include almotriptan, frovatriptan, naratriptan, sumatriptan, zolmitriptan, and a combination of ergotamine and caffeine anhydrous (hereafter referred to as ergotamine). Medications that are approved for migraine prophylaxis in Korea include amitriptyline, flunarizine, nadolol, propranolol, topiramate, and valproic acid/valproate/ divalproex (Appendix A). Furthermore, we classified each ingredient of the preventive therapy into the following drug classes: Amitriptyline was grouped under antidepressants, flunarizine under calcium channel blockers (CCB), nadolol and propranolol as beta-blockers, and topiramate and valproic acid/valproate/divalproex as anticonvulsants. If any preventive drug was prescribed at least once in 2017 , then we considered preventive medicine was utilized.

We evaluated the prescription pattern of migraine-specific acute therapy and preventive therapy. In the case of usage of multiple medications by a patient, the drug that had the highest number of DDD per month was regarded as the main acute therapy or main preventive therapy. Similarly, we summed the number of DDD per month for drugs belonging to a drug class, and the class with the highest number was considered the main drug class.

\section{Statistical analysis}

Categorical variables of the patient's baseline characteristics were expressed in frequencies and percentages using frequency analysis. To determine the association between prophylactic prescription and explanatory factors in candidates for migraine 
preventive therapy, we performed a chi-square test and multiple logistic regression analysis. Explanatory factors include demographic factors such as age group, sex, and insurance type; and health factors such as migraine type, migraine frequency, and comorbidities. The Hosmer-Lemeshow test was performed to check the fit of this model. We used the $\mathrm{R}$ Statistical Software (version 4.0.0; R Foundation for Statistical Computing, Vienna, Austria) to perform statistical analysis, and considered $p<0.05$ as statistically significant.

\section{Results}

\section{Characteristics of study subjects}

The number of patients requiring migraine prophylaxis was estimated as 534. The characteristics of study subjects and migraine prophylactic drug utilization are summarized in Table 1. Patients aged 50 years or older were the most among the three age groups (41.6\%). Female patients are almost 4 times more than male patients $(79.8 \%$ vs $20.2 \%)$. Most patients (96.3\%) were covered with NHI. Patients with MWoA was almost 5 times more than patients with MWA. For more than half of the patients, an appropriate migraine type was not assigned due to the limited code information. The most frequent comorbidity was dyslipidemia (36.9\%), followed by osteoarthritis $(30.0 \%)$, then depressive disorders (24.0\%).

\section{Preventive therapy utilization rate}

Of the 534 patients, 234 (43.8\%) were prescribed preventive therapy at least once in 2017 (Table 1). Migraine preventive drug use decreased with the advanced age $(p=0.382)$, and was lower in men than in women $(p=0.170)$. Further, migraine preventive therapy was higher in patients with epilepsy ( $p<$ $0.001)$, depressive disorders $(p<0.001)$, insomnia $(p=0.016)$, dyslipidemia $(p=0.013)$, and osteoarthritis $(p=0.038)$.

\section{Predictors of preventive therapy utilization}

Table 2 presents the results of the multiple logistic regression analysis. Patients aged 50 years or older were less likely to use preventive drugs than those under 40 years of age (odds ratio $[\mathrm{OR}]=0.513$; confidence interval $[\mathrm{CI}]=0.302$ 0.862). Among comorbidities, epilepsy was the most influencing factor $(\mathrm{OR}=7.326$; $\mathrm{CI}=2.586-26.488)$. Patients with depressive disorders are more than 2 times likely to utilize migraine preventive therapy $(\mathrm{OR}=2.439 ; \mathrm{CI}=1.567-3.827)$.

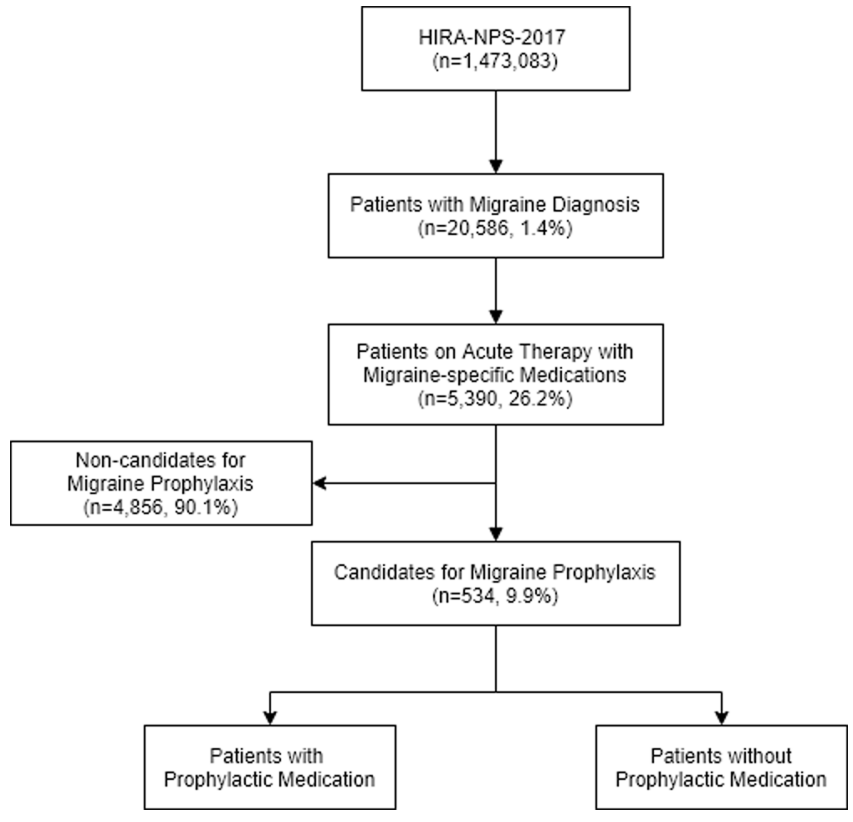

Fig. 1. Case extraction diagram. HIRA-NPS, health insurance review and assessment service-national patient sample

\section{Patterns of acute and preventive therapy for migraine}

Table 3 presents the patterns of drugs prescribed for patients who have an indication for migraine prophylaxis. Sumatriptan was the most frequently prescribed medication as an acute treatment $(29.0 \%)$, followed by ergotamine $(27.3 \%)$, naratriptan (19.1\%), almotriptan (10.1\%), zolmitriptan (8.8\%), and frovatriptan $(5.6 \%)$.

The most commonly used preventive drugs were propranolol $(35.0 \%)$, followed by flunarizine $(32.5 \%)$, topiramate $(13.7 \%)$, amitriptyline (12.8\%), valproic acid/valproate/divalproex (3.8\%), and nadolol $(2.1 \%)$. When preventive therapies were classified by the drug class, beta-blockers were the most prescribed (37.2\%), followed by CCB (32.5\%), anticonvulsants (17.5\%), and antidepressants $(12.8 \%)$.

\section{Discussion}

This study investigated the utilization of migraine preventive therapy in eligible patients, determined the factors influencing the use of preventive therapy, and analyzed the prescribing patterns of migraine-specific medications and preventive medications.

Patients requiring migraine prophylaxis therapy was about $10 \%$ of the migraine patients who had been prescribed acute therapy at least once $(n=5,390)$. This figure is low compared 
Table 1. Demographic characteristics and migraine prophylactic drug utilization.

\begin{tabular}{|c|c|c|c|c|}
\hline & & $\mathrm{N}$ & Prophylactic utilization (\%) & $p$-Value \\
\hline Total & & 534 & $234(43.8)$ & \\
\hline \multirow{3}{*}{ Age group } & $<40$ & $147(27.5)$ & $71(48.3)$ & 0.382 \\
\hline & $40-49$ & $165(30.9)$ & $72(43.6)$ & \\
\hline & $\geq 50$ & $222(41.6)$ & $91(41.0)$ & \\
\hline \multirow{2}{*}{ Sex } & Male & $108(20.2)$ & $41(38.0)$ & 0.170 \\
\hline & Female & $426(79.8)$ & 193(45.3) & \\
\hline \multirow{2}{*}{ Insurance } & NHI & $514(96.3)$ & $225(43.8)$ & 0.914 \\
\hline & MedAid/PVI & $20(3.7)$ & $9(45.0)$ & \\
\hline \multirow{3}{*}{ Migraine type } & Migraine without aura & $202(37.8)$ & $113(55.9)$ & $<0.001$ \\
\hline & Migraine with aura & $40(7.5)$ & $17(42.5)$ & \\
\hline & Other migraine & $292(54.7)$ & 104(35.6) & \\
\hline \multirow{2}{*}{ Epilepsy } & No & $508(95.1)$ & $212(41.7)$ & $<0.001$ \\
\hline & Yes & $26(4.9)$ & $22(84.6)$ & \\
\hline \multirow{2}{*}{ Depressive disorders } & No & $406(76.0)$ & $152(37.4)$ & $<0.001$ \\
\hline & Yes & $128(24.0)$ & $82(64.1)$ & \\
\hline \multirow{2}{*}{ Insomnia } & No & $464(86.9)$ & 194(41.8) & 0.016 \\
\hline & Yes & $70(13.1)$ & $40(57.1)$ & \\
\hline \multirow{2}{*}{ Hypertension } & No & $416(77.9)$ & $178(42.8)$ & 0.367 \\
\hline & Yes & $118(22.1)$ & $56(47.5)$ & \\
\hline \multirow{2}{*}{ Diabetes mellitus } & No & $466(87.3)$ & $198(42.5)$ & 0.105 \\
\hline & Yes & $68(12.7)$ & $36(52.9)$ & \\
\hline \multirow{2}{*}{ Dyslipidemia } & No & $337(63.1)$ & $134(39.8)$ & 0.013 \\
\hline & Yes & 197(36.9) & $100(50.8)$ & \\
\hline \multirow{2}{*}{ Osteoarthritis } & No & $374(70.0)$ & $153(40.9)$ & 0.038 \\
\hline & Yes & $160(30.0)$ & $81(50.6)$ & \\
\hline \multirow{2}{*}{ Rheumatoid arthritis } & No & $507(94.9)$ & $222(43.8)$ & 0.947 \\
\hline & Yes & $27(5.1)$ & $12(44.4)$ & \\
\hline \multirow{2}{*}{ Asthma } & No & $458(85.8)$ & $194(42.4)$ & 0.095 \\
\hline & Yes & $76(14.2)$ & $40(52.6)$ & \\
\hline \multirow{2}{*}{ Thyroid gland disorders } & No & $434(81.3)$ & $190(43.8)$ & 0.968 \\
\hline & Yes & $100(18.7)$ & $44(44.0)$ & \\
\hline
\end{tabular}

NHI, national health insurance; MedAid, medical aid; PVI, patriots \& veterans insurance; COPD, chronic obstructive pulmonary disease

to $38.8 \%$ of migraine patients who are eligible for migraine prophylaxis in the US. ${ }^{2)}$ The claim data we used did not identify the use of over-the-counter (OTC) drugs when estimating migraine days with the monthly average number of DDD of acute therapy. Since a significant number of migraine sufferers $(49 \%)$ use OTC pain medications, ${ }^{15)}$ those who are candidates for migraine preventive treatment might have been underestimated in our study.

In this study, $43.8 \%$ of patients had been prescribed preventive therapy at least once in 2017 , and the proportion was similar or higher than in other countries. A study in the US reported that about $39 \%$ of migraineurs had an indication for migraine prophylaxis, but only $32 \%$ of them received preventive therapy. ${ }^{2)}$ Caetano et al. found that among 77 
Table 2. Multiple logistic regression analysis of migraine prophylactic drug utilization

\begin{tabular}{|c|c|c|c|c|}
\hline & & Odds Ratio (OR) & $95 \% \mathrm{CI}$ & $p$-Value \\
\hline \multicolumn{5}{|l|}{ Total $(\mathrm{N}=534)$} \\
\hline \multirow{3}{*}{ Age group } & $<40$ & Reference & & \\
\hline & $40-49$ & 0.695 & $0.425-1.133$ & 0.146 \\
\hline & $\geq 50$ & 0.513 & $0.302-0.862$ & 0.012 \\
\hline \multirow{2}{*}{ Sex } & Male & Reference & & \\
\hline & Female & 1.606 & $0.988-2.649$ & 0.059 \\
\hline \multirow{2}{*}{ Insurance type } & NHI & Reference & & \\
\hline & MedAid/PVI & 0.608 & $0.198-1.736$ & 0.362 \\
\hline \multirow{3}{*}{ Migraine type } & Migraine without aura & Reference & & \\
\hline & Migraine with aura & 0.657 & $0.310-1.371$ & 0.266 \\
\hline & Other migraine & 0.471 & $0.316-0.700$ & $<0.001$ \\
\hline \multirow{10}{*}{ Comorbidities } & Epilepsy & 7.326 & $2.586-26.488$ & $<0.001$ \\
\hline & Depressive disorders & 2.439 & $1.567-3.827$ & $<0.001$ \\
\hline & Insomnia & 1.266 & $0.709-2.265$ & 0.425 \\
\hline & Hypertension & 1.085 & $0.640-1.834$ & 0.761 \\
\hline & Diabetes mellitus & 1.119 & $0.580-2.163$ & 0.736 \\
\hline & Dyslipidemia & 1.532 & $0.947-2.488$ & 0.083 \\
\hline & Osteoarthritis & 1.467 & $0.951-2.268$ & 0.083 \\
\hline & Rheumatoid arthritis & 1.359 & $0.553-3.282$ & 0.496 \\
\hline & Asthma & 1.226 & $0.712-2.107$ & 0.460 \\
\hline & Thyroid gland disorders & 0.687 & $0.406-1.149$ & 0.157 \\
\hline \multicolumn{2}{|c|}{$c$-statistic } & \multicolumn{2}{|c|}{0.713} & \\
\hline \multicolumn{2}{|c|}{$p$ value of Hosmer-Lemeshow test } & \multicolumn{2}{|c|}{0.703} & \\
\hline
\end{tabular}

NHI, national health insurance; MedAid, medical aid; PVI, patriots \& veterans insurance; COPD, chronic obstructive pulmonary disease

patients who were eligible for prophylactic treatment, only $22 \%$ of them were on preventive treatment in Portugal. ${ }^{16)} \mathrm{We}$ might have overestimated the preventive drug utilization rate since we considered a patient as a preventive drug user if he/ she was prescribed the index drug at least once a year. Nevertheless, more than half of the patients had not received optimal preventive therapy in Korea.

One previous study found that women experienced more frequent migraine headaches, greater disability, and more frequent consultation with their physicians about their headaches. ${ }^{17,18)}$ The ratio of women to men in the population of candidates for migraine prophylaxis was $4: 1$, which differed in frequency by age groups. However, there was no significant relationship between sex and the use of preventive treatment in our study. Common comorbidities that occur with migraine and affect the treatment include depression, anxiety disorders, epilepsy, sleep disorders, stroke, and other pain disorders. ${ }^{19)}$ Among various comorbidities, the most frequent comorbid conditions according to the results of our study are dyslipidemia (36.9\%), osteoarthritis $(30.0 \%)$, depressive disorders $(24.0 \%)$, and hypertension $(22.1 \%)$.

Diamond et al. reported that female and elderly patients with migraine were more likely engaged with current or past utilization of preventive therapy. ${ }^{15)}$ On the other hand, there was no significant relationship between sex and prescription of preventive agents according to a Netherlands study. ${ }^{20)} \mathrm{We}$ found that women received more preventive treatment, although it was not statistically significant, and preventive drug prescriptions decreased in older people aged over 50 years. This may be due to the high use of multiple medications by older patients and physicians might be concerned about adverse drug reactions, drug-drug interactions, or drug-disease 
Table 3. Patterns of acute and preventive therapy in patients eligible for migraine prophylaxis

\begin{tabular}{lcc}
\hline & $\mathrm{N}$ & Drug utilization (\%) \\
\hline Drug for acute therapy & 534 & 100.0 \\
Sumatriptan & 155 & 29.0 \\
Ergotamine & 146 & 27.3 \\
Naratriptan & 102 & 19.1 \\
Almotriptan & 54 & 10.1 \\
Zolmitriptan & 47 & 8.8 \\
Frovatriptan & 30 & 5.6 \\
\hline Drug for preventive therapy & 234 & 100.0 \\
Propranolol & 82 & 35.0 \\
Flunarizine & 76 & 32.5 \\
Topiramate & 32 & 13.7 \\
Amitriptyline & 30 & 12.8 \\
Valproic acid/valproate/divalproex & 9 & 3.8 \\
Nadolol & 5 & 2.1 \\
\hline Drug class of preventive therapy & 234 & 100.0 \\
Beta-blockers & 87 & 37.2 \\
Calcium channel blockers & 76 & 32.5 \\
Anticonvulsants & 41 & 17.5 \\
Antidepressants & 30 & 12.8 \\
\hline
\end{tabular}

interactions. $^{20,21)}$

Caetano et al. reported that patients with MWA had higher rates of migraine prophylaxis compared to the patients with MWoA. ${ }^{16)}$ However, our results showed the opposite trend. We were not able to draw any meaningful hypothesis from this finding since more than half of the patients were not assigned to any specific migraine subtype.

More preventive agents were prescribed in the presence of epilepsy and depressive disorder as comorbidities. This may be due to the fact that the approved migraine prophylaxis agents also have an indication of epilepsy or depression. ${ }^{22)}$ In particular, depression is associated with severe migraines, and frequent migraine headaches with high severity are linked to increased use of migraine prevention treatments in these conditions. $^{20,23)}$

Sumatriptan was the most frequently prescribed drug for acute treatment, followed by ergotamine. According to a study carried out in the US, sumatriptan was the most commonly used drug for acute treatment, followed by rizatriptan. ${ }^{11)}$ Meanwhile, Sheftell et al. found that the most frequently prescribed drug was sumatriptan, followed by zolmitriptan, and then rizatriptan. ${ }^{24)}$ In comparison, at the time of this research, rizatriptan was not available in Korea and thereby excluded from the analysis. Since we analyzed the frequency of prescriptions by considering the highest average number of DDD per month as the main acute therapy in each patient, the results may differ due to differences in the methodology between the studies.

According to the Canadian Headache Society guideline and the European Federation of Neurological Societies (EFNS) guideline, ergotamine is not recommended for acute migraine attacks because it has lower efficacy and greater risk of side effects than triptans. ${ }^{25,26)}$ However, ergotamine was the second most frequently used medication after sumatriptan in Korea. This is probably due to the cost advantage of ergotamine. Triptans were about 50 to 80 times more expensive than ergotamine at the time of this study.

The most commonly prescribed preventive medicine was propranolol, followed by flunarizine, and topiramate. In terms of drug classes, beta-blockers were the most commonly used ones. The most frequently prescribed preventive drug in a US study was topiramate. ${ }^{11)}$ Another study found that betablockers were the most frequently used preventive drug as well as the first choice of physicians followed by TCA, CCB, anticonvulsant (topiramate) in that order. ${ }^{27)}$

According to the international guidelines of the $\mathrm{WHO} / \mathrm{EHF}$, beta-blockers, topiramate, flunarizine, sodium valproate, amitriptyline are effective for migraine prevention and are recommended for use as a first tier. ${ }^{28)}$ The results of our study suggest that the medications were used according to the guidelines. Hypertension is highly prevalent in Korea as well as worldwide. $^{29)}$ The high prevalence of hypertension may be related to the frequent prescription of beta-blockers and CCB as migraine prophylactic medication. The choice of migraine preventive therapy should take into account not only the efficacy of the drug but also safety, tolerability, patient preferences, and comorbidities. ${ }^{4,30)}$

There are several limitations to our study. First, we used insurance claim data, originally generated for reimbursement purposes. Considering that it is not collected for research purposes, diagnostic information may be inaccurate. For example, it is difficult to compare patients with aura and without aura because migraine-type was not accurately classified and many patients were assigned to "other migraines group". Second, the claim data did not provide detailed 
clinical information of the patient such as migraine severity, exact migraine frequency, medication tolerability, and adverse drug reactions. In addition, socioeconomic characteristics such as economic status, education, or health behavior were not available. Third, since the migraine days per month were replaced by the monthly average number of DDDs of acute therapy, the exact migraine days experienced by the patient could not be determined. Furthermore, when counting the number of DDD, it could have been underestimated because OTC drugs were excluded from the analysis. Fourth, it was not known whether patients were prescribed these drugs to prevent migraines or to treat other comorbid conditions.

Despite these limitations, our study provides evidence that migraine prevention therapy is underutilized in Korea. We also identified influencing factors that could predict the use of migraine prevention medications and compared the pattern of drug prescriptions with other countries. Our findings warrant further research on a reason why patients who meet the criteria for migraine prophylaxis are not receiving adequate preventive therapy.

\section{Conclusions}

In conclusion, more than half of the eligible patients with migraine were not currently receiving prophylaxis. Positive factors affecting the use of migraine prophylaxis were epilepsy, and depressive disorders. The most commonly prescribed acute therapy for migraine relief was sumatriptan, and preventive therapy was propranolol.

\section{Acknowledgment}

We used HIRA-NPS-2017-0014 data from the Health Insurance Review and Assessment Service for this study, but the results have no connection with the Ministry of Health and Welfare or HIRA.

\section{Conflict of Interest}

We declare no conflict of interests.

\section{References}

1. GBD 2016 Disease and Injury Incidence and Prevalence Collaborators. Global, regional, and national incidence, prevalence, and years lived with disability for 328 diseases and injuries for 195 countries, 1990-2016: a systematic analysis for the Global Burden of Disease Study 2016. Lancet 2017;390(10100):1211-59.

2. Lipton RB, Bigal ME, Diamond M, et al. Migraine prevalence, disease burden, and the need for preventive therapy. Neurology 2007;68(5):343-9.

3. Kim BK, Chu MK, Lee TG, Kim JM, Chung CS, Lee KS. Prevalence and impact of migraine and tension-type headache in Korea. J Clin Neurol 2012;8(3):204-11.

4. Lipton RB, Silberstein SD. Episodic and chronic migraine headache: breaking down barriers to optimal treatment and prevention. Headache 2015;55 Suppl 2:103-22; quiz 123-6.

5. Headache Classification Committee of the International Headache Society (IHS). The international classification of headache disorders, 3rd edition. Cephalalgia 2018;38(1):1-211.

6. Silberstein SD, Winner PK, Chmiel JJ. Migraine preventive medication reduces resource utilization. Headache 2003;43(3):1718.

7. Lee K-S. The diagnosis and most-updated therapy of migraine. J Korean Med Assoc 2009;52(5):500-6.

8. Marmura MJ, Silberstein SD, Schwedt TJ. The acute treatment of migraine in adults: The American headache society evidence assessment of migraine pharmacotherapies. Headache 2015;55(1):320.

9. Tfelt-Hansen PC. Evidence-based guideline update: pharmacologic treatment for episodic migraine prevention in adults: report of the Quality Standards subcommittee of the American Academy of Neurology and the American Headache Society. Neurology 2013; 80(9):869-70.

10. Kim L, Kim JA, Kim S. A guide for the utilization of health insurance review and assessment service national patient samples. Epidemiol health 2014;36:e2014008.

11. Ford JH, Jackson J, Milligan G, Cotton S, Ahl J, Aurora SK. A realworld analysis of migraine: A cross-sectional study of disease burden and treatment patterns. Headache 2017;57(10):1532-44.

12. Vo P, Gao W, Zichlin ML, et al. Real-world healthcare resource utilization related to migraine treatment failure: a panel-based chart review in France, Germany, Italy, and Spain. J Med Econ 2019; 22(9):953-9.

13. Piccinni C, Cevoli S, Ronconi G, et al. A real-world study on unmet medical needs in triptan-treated migraine: prevalence, preventive therapies and triptan use modification from a large Italian population along two years. J Headache Pain 2019;20(1):74.

14. Devine J, Hadsall R, Cline R, Schommer J, Virnig B. Effect of daily migraine prevention on health care utilisation in an insured patient population. J Headache Pain 2007;8(2):105-13.

15. Diamond S, Bigal ME, Silberstein S, Loder E, Reed M, Lipton RB. Patterns of diagnosis and acute and preventive treatment for migraine in the United States: results from the American Migraine Prevalence and Prevention study. Headache 2007;47(3):355-63.

16. Caetano A, Ladeira F, Mendonca M, et al. Underuse of prophylactic treatment among Portuguese patients with primary headache: A retrospective observational study. J Oral Facial Pain Headache 2019;33(3):331-6.

17. Lipton RB, Stewart WF, Simon D. Medical consultation for migraine: results from the American Migraine Study. Headache 1998;38(2):87-96.

18. Steiner T, Scher A, Stewart W, Kolodner K, Liberman J, Lipton R. The prevalence and disability burden of adult migraine in England 
and their relationships to age, gender and ethnicity. Cephalalgia 2003;23(7):519-27.

19. Silberstein SD, Dodick D, Freitag F, et al. Pharmacological approaches to managing migraine and associated comorbidities-clinical considerations for monotherapy versus polytherapy. Headache 2007;47(4):585-99.

20. Rahimtoola H, Buurma H, Tijssen CC, Leufkens HG, Egberts AC. Incidence and determinants of migraine prophylactic medication in the Netherlands. Eur J Clin Pharmacol 2002;58(2):149-55.

21. Passmore AP, Crawford VLS, Beringer TRO, Gilmore DH, Montgomery A. Determinants of drug utilization in an elderly population in North and West Belfast. Pharmacoepidemiol Drug Saf 1995;4(3):147-60.

22. Silberstein SD. Comprehensive management of headache and depression. Cephalalgia 1998;18 Suppl 21:50-5.

23. Radat F, Sakh D, Lutz G, el Amrani M, Ferreri M, Bousser MG Psychiatric comorbidity is related to headache induced by chronic substance use in migraineurs. Headache 1999;39(7):477-80.

24. Sheftell FD, Feleppa M, Tepper SJ, Volcy M, Rapoport AM, Bigal ME. Patterns of use of triptans and reasons for switching them in a tertiary care migraine population. Headache 2004;44(7):661-8.

25. Worthington I, Pringsheim T, Gawel MJ, et al. Canadian Headache Society Guideline: acute drug therapy for migraine headache. Can J Neurol Sci 2013;40(5 Suppl 3):S1-S80.

26. Evers S, Afra J, Frese A, et al. EFNS guideline on the drug treatment of migraine--revised report of an EFNS task force. Eur J Neurol 2009;16(9):968-81.

27. Tepper S, D'Amico D, Baos V, Blakeborough P, Dowson A. Guidelines for prescribing prophylactic medications for migraine: A survey among headache specialist physicians in different countries. Headache Care 2004;1(4):267-72.

28. Steiner TJ, Martelletti P. Aids for management of common headache disorders in primary care. J Headache Pain 2007;8 Suppl 1:S2.

29. Kim Y, Suh YK, Choi H. BMI and metabolic disorders in South Korean adults: 1998 Korea National Health and Nutrition Survey. Obes Res 2004;12(3):445-53.

30. D'Amico D, Lanteri-Minet M. Migraine preventive therapy: Selection of appropriate patients and general principles of management. Expert Rev Neurother 2006;6(8):1147-57. 


\section{Appendix A. Disease and Drug Codes}

\section{Disease and Drug Codes (http://koicd.kr/)}

\begin{tabular}{|c|c|}
\hline Disease (KCD-7) & \\
\hline Migraine without aura & G430 \\
\hline Migraine with aura & G431 \\
\hline Other migraines & G432, G433, G438, G439 \\
\hline Hypertension & $\mathrm{I} 10-13$ \\
\hline Epilepsy & G40-41 \\
\hline Schizophrenia & $\mathrm{F} 20$ \\
\hline Depressive disorders & F32-33 \\
\hline Anxiety disorders & F064, F40, F410-413, F418, F419 \\
\hline Sleep disorders & F51, G47 \\
\hline Insomnia & F510, G470 \\
\hline Diabetes mellitus & E10-14 \\
\hline Osteoarthritis & M15-19 \\
\hline Rheumatoid arthritis & M05, M06, M080, M120, M123 \\
\hline Asthma & J45-46 \\
\hline Chronic obstructive pulmonary disease & $\mathrm{J} 44$ \\
\hline Malaise and fatigue & $\mathrm{R} 53$ \\
\hline Disorders of thyroid gland & E00-07 \\
\hline Dyslipidemia & E78 \\
\hline Obesity & E66 \\
\hline \multicolumn{2}{|l|}{ Drug } \\
\hline Sumatriptan & 233802ATB, 233803ATB \\
\hline Naratriptan & 415501ATB \\
\hline Zolmitriptan & 415601ATB \\
\hline Almotriptan & 499401ATB \\
\hline Frovatriptan & 509501ATB \\
\hline Ergotamine/caffeine anhydrous & 251800ATB \\
\hline Amitriptyline & $107501 \mathrm{ATB}, 107502 \mathrm{ATB}, 107504 \mathrm{ATB}$ \\
\hline Topiramate & $\begin{array}{l}\text { 241801ACR, 241801ATB, 241802ACR, 241803ACH, 241803ATB, } \\
\text { 241804ACH, 241804ATB }\end{array}$ \\
\hline Valproic acid/valproate/divalproex & $\begin{array}{l}\text { 246901ATE, 246902ATE, 246903ATE, 247001ACS, 247002ACS, } \\
\text { 229701ACR, 229701ATR, 229703ATB, 229705ATB, 229705ATR, } \\
\text { 229706ATR, 229707ATR, 147701ATE, } 147702 \text { ATR, } 147801 \text { ACH }\end{array}$ \\
\hline Propranolol & 219901AТВ, 219904AТВ \\
\hline Nadolol & 198301ATB \\
\hline Flunarizine & $160501 \mathrm{ACH}$ \\
\hline
\end{tabular}

KCD; Korean Classification of Diseases 